\title{
The great oxidation of Earth's atmosphere
}

\author{
Zdzislaw E. Musielak ${ }^{1}$, Manfred Cuntz ${ }^{1}$ and Dipanjan Roy ${ }^{2}$ \\ Department of Physics, University of Texas at Arlington, Arlington, TX 76019, USA \\ email: zmusielak@uta.edu, cuntz@uta.edu \\ ${ }^{2}$ Institut des Sciences du Mouvement UMR CNRS 6233, Université de la Méditerranée, 13288 \\ Marseille, France \\ email: dipanjan.roy@etumel.univmed.fr
}

\begin{abstract}
A simplified model of the Earth's atmosphere consisting of three nonlinear differential equations with a driving force was developed by Goldblatt et al. (2006). They found a steadystate solution that exhibits bistability and identified its upper value with the great oxidation of the Earth's atmosphere. Noting that the driving force in their study was a step function, it is the main goal of this paper to investigate the stability of the model by considering two different more realistic driving forces. The stability analysis is performed by using Lyapunov exponents. Our results show that the model remains stable and it does not exhibit any chaotic behavior.
\end{abstract}

\section{Introduction}

There is strong geological evidence that the oxygen concentration in the Earth's atmosphere increased from $10^{-5}$ of its present atmospheric level (PAL) to $10^{-2} \mathrm{PAL}$ (the so-called great oxidation) about 2.4 Gyr ago (Catling et al. 2001). To explain this phenomenon, Goldblatt et al. (2006) developed a simplified atmospheric model in which the time evolution of oxygen, methane and carbon is driven by the sudden decline of reductants to the surface.

Mathematically, the model is described by a set of three nonlinear differential equations with a driving force. Goldblatt et al. (2006) approximated the driving force by a step function and obtained a steady-state solution that exhibits bistability. They identified the high value of oxygen in this bistability as the great oxidation of the Earth's atmosphere. In this paper, we consider the same mathematical model but with two different and more realistic driving forces. We investigate stability by using Lyapunov exponents. Our results show that the model is stable and does not exhibit chaos.

\section{Results and discussion}

The set of three nonlinear equations that describes the time evolution of oxygen, methane and carbon is discussed in detail by Goldblatt et al. (2006) and Cuntz et al. (2009), and it will not be repeated here. However, we want to emphasize that the explicit dependence on time of the driving force makes the set of equations to be non-autonomous. A standard method to perform stability analysis of such a system of equations is to investigate its Lyapunov exponents. The fact that the Lyapunov exponent method is a valuable tool for studying dynamical systems with bistability has been shown in the literature (Badzey \& Mohanty 2005).

The number of Lyapunov exponents for a dynamical system is the same as the number of dependent variables representing the system. However, if the system is nonautonomous, then one additional Lyapunov exponent must be added (Wu et al. 2007). For the considered model, the equations describing the time evolution of oxygen and 

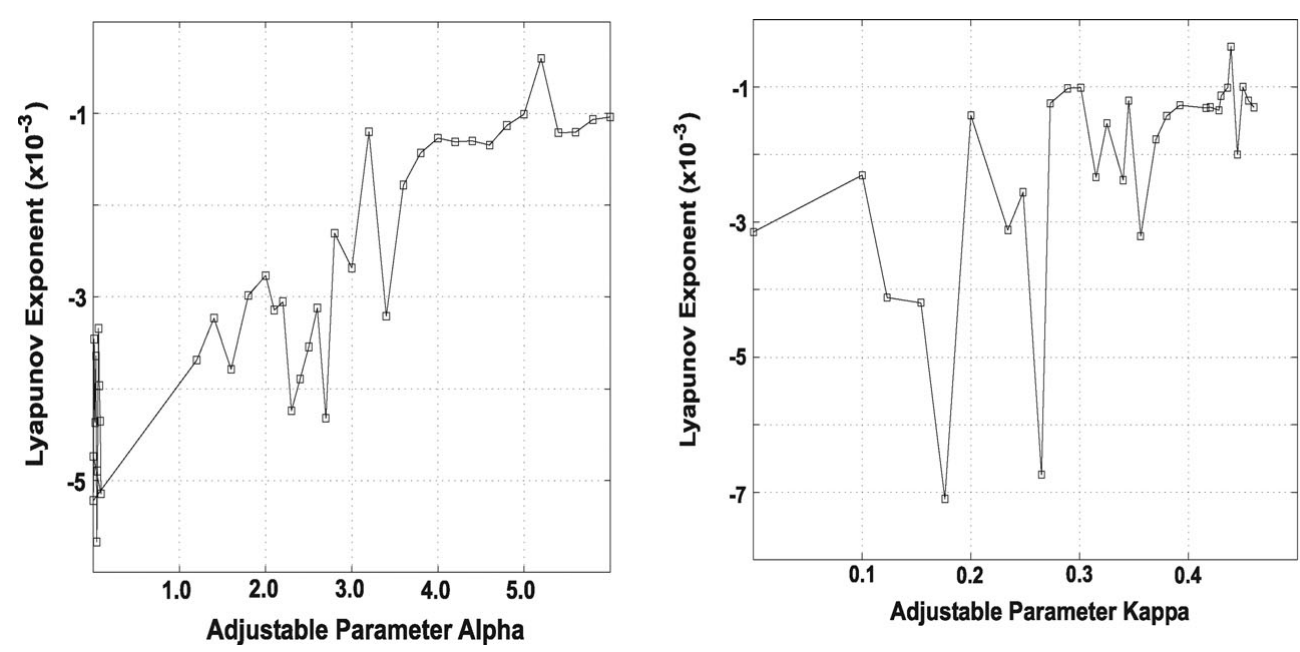

Figure 1. Lyapunov exponent for methane is plotted versus the parameters $\alpha$ and $\kappa$ of the two driving forces used in our numerical calculations.

methane are mutually dependent and non-autonomous, which means that three Lyapunov exponents are needed to investigate the stability of this subset of two equations. Note that the equation describing the time evolution of carbon is independent and the corresponding Lyapunov exponent is zero (Cuntz et al. 2009).

To determine the stability of the model, we consider two driving forces. The so-called exponential driving force is given by $r(t)=r_{0} e^{-\alpha t / t_{0}}$ and the logistic driving force is given by $r(t)=r_{0} \kappa^{-t / t_{0}}$ with $t_{0}=10^{7}$ yrs and $\alpha$ and $\kappa$ as parameters. The system equations are solved numerically by implementing one of these driving forces. Thereafter, the resulting three Lyapunov exponents are calculated. Since two of the exponents (oxygen and methane) are negative, and the third one corresponding to the explicit time dependency of the system is zero, the system can be classified as periodic (Hilborn 1994). The Lyapunov exponent for methane calculated for the two driving forces is plotted in Fig. 1. It shows that the exponent remains negative for all values of $\alpha$ and $\kappa$ considered.

\section{Conclusions}

We studied the model of the Earth's atmosphere developed by Goldblatt et al. (2006) by using two different driving forces. Our results show that the model remains stable and it does not exhibit any chaotic behavior for the considered range of parameters. If confirmed by further studies, this result carries important implications for the biochemistry of early Earth as discussed by Kasting (2006) and others, and may be of interest for the investigation of Earth-type exoplanets as well.

\section{References}

Badzey, R. L. \& Mohanty, P. 2005, Nature 437, 995

Catling, D. C., Zahnle, K. J., \& McKay, C. P. 2001, Science 293, 839

Cuntz, M., Roy, D., \& Musielak, Z. E. 2009, ApJ (Letters) 706, L178

Goldblatt, C., Lenton, T. M., \& Watson, A. J. 2006, Nature 443, 683

Hilborn, R. C. 1994, Chaos and Nonlinear Dynamics, (Oxford: Oxford University Press)

Kasting, J. F. 2006, Nature 443, 643

Wu, X., Wang, J., Lu, J., \& Iu, H. H. C. 2007, Chaos, Solitons \& Fractals 32, 1483 